\title{
Composition en acides gras poly-insaturés de notre assiette et utilisation des matières premières agricoles en France : une amélioration lente, mais insuffisante
}

\author{
Michel Duru ${ }^{1,2, *}$ et Marie-Benoît Magrini ${ }^{1,2}$ \\ 1 INRA, UMR 1248 AGIR, 31326 Castanet Tolosan, France \\ 2 Université Toulouse, INPT, UMR AGIR, 31029 Toulouse, France
}

Reçu le 8 novembre 2016 - Accepté le 10 février 2017

\begin{abstract}
Résumé - Un consensus est établi qu'un apport insuffisant en acide alpha-linolénique (ALA) et un rapport LA (acide linoléique)/ALA trop élevé contribuent à un accroissement de nombreuses maladies chroniques. Pour évaluer l'évolution de ces apports en France au cours des 20 dernières années (1993-2012), nous estimons les quantités apparentes consommées incluant la composition des produits animaux selon leur mode d'alimentation. Nous montrons que les consommations en LA et ALA ont respectivement diminué et augmenté (de 0,72 à $0,94 \mathrm{~g} / \mathrm{j}$ pour l'ALA) et que le rapport LA/ALA est passé de 18,3 à 12,4 . L'évolution observée est cohérente avec celle de la composition du lait maternel. Ces valeurs sont proches de celles obtenues en 2006 par enquêtes auprès d'un échantillon de consommateurs. Elle est discutée au regard des politiques publiques de santé, des stratégies de production agricole et du comportement du consommateur. Ces lentes évolutions suggèrent que des changements radicaux restent nécessaires pour se rapprocher des recommandations $(\mathrm{ALA}=1,8 \mathrm{~g} / \mathrm{j} ; \mathrm{LA} / \mathrm{ALA} \leq 4)$. Elles questionnent les marges de manœuvre dont dispose le consommateur pour choisir des produits animaux selon leur mode d'alimentation et les huiles alimentaires selon leurs apports.
\end{abstract}

Mots clés : AGPI/consommation apparente / huiles végétales / prairie / produits animaux / tourteaux

\begin{abstract}
Polyunsaturated fatty acids composition of our meals and use of agricultural raw products in France: a slow improvement, but not sufficient. A consensus is established that an insufficient intake of alpha-linolenic acid (ALA) and a ratio LA (linoleic acid)/ALA too high contribute to an increase in chronic diseases. To assess the evolution of their intake during the last 20 years (1993-2012) in France, we estimate their apparent consumption, taking into account the composition of animal products according to their feeding regimes. We show that the consumption of LA and ALA have decreased and increased respectively (from 0.72 to $0.94 \mathrm{~g} / \mathrm{d}$ for ALA) and the ratio LA/ALA increased from 18.3 to 12.4. This trend is consistent with that of the breast milk composition. These values are close to those obtained in 2006 from surveys of a sample of consumers. It is discussed in terms of public health policies, agricultural production strategies and consumer behavior. These slow changes suggest that radical ones are needed to meet recommendations $(\mathrm{ALA}=1.8 \mathrm{~g} / \mathrm{d} ; \mathrm{LA} / \mathrm{ALA} \leq 4)$. They question the room for the consumer to choose animal products according to their feeding regimes and oils according to their composition.
\end{abstract}

Keywords: animal products / apparent consumption / grassland / oil cake / vegetable oils / PUFA

\section{Introduction}

Les impacts de l'agriculture et de l'agro-alimentaire sur la santé humaine sont fortement médiatisés pour des enjeux phares comme ceux liés à l'usage des pesticides. En revanche, une attention moindre a été accordée aux acides gras polyinsaturés (AGPI) dont les apports et équilibres dépendent

\footnotetext{
* Correspondance : michel. duru@inra.fr
}

beaucoup des modes d'alimentation des animaux d'élevage, des matières grasses utilisées par l'industrie agroalimentaire et des huiles utilisées par le consommateur. Nombre d'auteurs attirent l'attention sur les enjeux de santé publique liés à la modification en profondeur du profil en acides gras de l'assiette dans les pays occidentaux, du fait des changements progressifs de pratiques agricoles, de processus industriels et de modes de consommation depuis la moitié du $20^{\mathrm{e}}$ siècle (Molendi-Coste et al., 2011; Simopoulos, 2007). 
La famille des oméga-3 regroupe notamment l'acide alphalinolénique $\left(\operatorname{ALA} *^{1}\right)$, précurseur de l'acide eicosapentaénoïque $(\mathrm{EPA} *)$ et de l'acide docosahexaénoïque (DHA $*$ ). L'acide linoléique $(\mathrm{LA} *)$ est le précurseur des oméga-6 à longues chaînes dont l'acide arachidonique (ARA $*)$. Le LA, l'ALA sont indispensables car nous ne pouvons les produire, de même que le DHA du fait de sa faible formation par conversion de l'ALA. Les médiateurs lipidiques générés à partir des AGPI à longues chaînes (''ARA, l'EPA et le DHA) ont des rôles importants dans la régulation des états inflammatoires. L'un des rôles des oméga-3 est de contrarier les activités pro-inflammatoires des oméga-6 (Simopoulos, 2007). C'est pourquoi le déséquilibre entre LA et ALA est préjudiciable à la synthèse et à la disponibilité des acides gras EPA et DHA, d'autant que les apports en EPA et DHA sont généralement inférieurs aux recommandations (Anses, 2015). Le rapport LA/ALA de notre alimentation joue donc un rôle essentiel.

Concernant les relations entre AGPI et maladies chroniques, les travaux les plus anciens portent sur les maladies cardiovasculaires: essais cliniques (Buxton et al., 2004); méta-analyse (Delgado-Lista et al., 2011). Il a été montré que les oméga-3 exercent des actions antioxydantes, anti-inflammatoires et anti-thrombotiques et retardent le développement de plaques d'athérome (Colussi et al., 2017). La mise en évidence d'un lien avec la fréquence de certains cancers est difficile du fait d'incertitudes sur les données et de confusion d'effets dans les études épidémiologiques ; néanmoins certains auteurs concluent à l'intérêt de régimes enrichis en oméga-3 pour en réduire le risque (Pottel et al., 2013). Concernant les dépressions (Kiecolt-Glaser et al., 2012) et les troubles de l'humeur (Rosenblat et al., 2014), un effet significatif d'une insuffisance d'oméga-3 a été montré. À partir d'études épidémiologiques et d'essais cliniques, une méta-analyse a mis en évidence une relation effective, bien que limitée, entre le rapport oméga-6/-3 et le déclin cognitif (Loef et Walach, 2013). D'autres recherches ont montré un effet significatif de l'excès d'oméga-6 sur l'obésité de la souris (Ailhaud et al., 2006). Plus récemment, il a été montré l'effet de l'insuffisance d'oméga-3 sur certaines maladies liées au fonctionnement du cerveau, la maladie de Parkinson (Bousquet et al., 2011) et la DMLA sauf en présence d'un excès d'oméga-6 (Lecerf, 2010).

En 2000, les apports apparents de LA et ALA (sans tenir compte des pertes et gaspillages) sont estimés à 21 et $0,9 \mathrm{~g} / \mathrm{j}$ $(\mathrm{LA} / \mathrm{ALA}=23)$ pour la population française dans sa globalité, ce qui correspond depuis 1960 à une augmentation du LA $(\times 2,5)$ et une réduction de l'ALA $(\times 0,6)$ (Ailhaud et al., 2006). Selon l'Anses (2011), l'apport minimum physiologique est de $2 \%$ et $0,8 \%$ des apports énergétiques respectivement pour le LA et l'ALA, soit 4,4 et $1,8 \mathrm{~g} / \mathrm{j}$ pour un apport énergétique de $2000 \mathrm{kcal}$. Les apports recommandés (ANC) ont été fixés à $4 \%$ et $1 \%$ des $\mathrm{AE}$, soit $8,7 \mathrm{~g} / \mathrm{j}$ et $2,2 \mathrm{~g} / \mathrm{j}$ (Anses, 2011), ce qui correspond à ceux des adultes et des adolescents représentant $88 \%$ de la population française en 2012. Le rapport LA/ALA recommandé a été fixé à 4 (Anses, 2011), voire à une valeur inférieure (2) (Yang et al., 2016) pour un effet protecteur contre certaines maladies. Les recommandations en DHA, et EPA du fait de l'insuffisance en ALA dans notre alimentation, ont été fixées à $250 \mathrm{mg} / \mathrm{j}$ pour chacun.

\footnotetext{
${ }^{1}$ Abréviation de l'anglais.
}

Face à ce décalage alarmant entre recommandations et consommations observées, les organismes en charge de la santé publique ont cherché à sensibiliser l'ensemble des acteurs, notamment ceux de l'agroalimentaire et à les accompagner dans un dispositif partenarial et incitatif (Rapport lipides du PNNS, 2009).

L'objectif de cette étude est d'évaluer l'évolution des quantités de LA et d'ALA consommées en France au cours des deux dernières décades, pour savoir si l'écart aux recommandations s'est réduit ou non depuis le début des années 1990, et de comprendre quelles sont les raisons majeures de cette évolution, tout particulièrement au regard du choix des matières premières agricoles utilisées pour l'alimentation animale et humaine. Il s'agit aussi de comprendre si les évolutions observées peuvent être en lien avec le comportement des consommateurs ou si elles sont la conséquence de l'évolution des réglementations. Pour ce travail, nous nous sommes limités aux évolutions des apports de LA et ALA par les huiles végétales et les produits animaux pour lesquels nous avons tenu compte de l'évolution de la composition de l'alimentation des animaux (Mourot, 2015). Nous n'avons pas tenu compte des légumes et des fruits, qui contribuent à environ $7 \%$ et $11,7 \%$ des apports de LA et d'ALA (Anses, 2015).

\section{Méthode}

À partir des statistiques annuelles de la comptabilité nationale sur les flux économiques (production + importations-exportations), nous avons calculé les quantités consommées en AGPI, desquelles nous avons soustrait une estimation des pertes. Ces pertes concernent les déchets (peaux, os, arêtes pour les viandes et les poissons), la fraction non consommée des huiles utilisées pour la conserverie et la préparation des aliments, mais aussi le gaspillage alimentaire. L'incertitude étant forte pour les huiles non consommées, nous avons considéré plusieurs hypothèses.

Si les estimations annuelles de consommation apparente permettent d'obtenir des données continues d'évolution, elles restent empreintes d'incertitudes (pour l'estimation des pertes) et de simplifications (non prise en compte des modes de cuisson, composition moyenne en AGPI pour la viande). Aussi, nous avons comparé nos données à celles des estimations directes de consommation issues d'enquêtes réalisées en 2006 (Anses, 2015) et 2010 (Ferry, com pers), et à celles des teneurs du lait maternel (Combe et Vaysse, 2011; Couëdelo et al., 2014) qui est un marqueur fiable à long terme de la consommation en AGPI des femmes allaitantes.

\subsection{Huiles et graisses végétales}

Les principales huiles végétales utilisées pour l'assaisonnement, la cuisine (cuisson, friture), la conserverie, mais aussi pour la fabrication d'une diversité de produits (pâtisseries, margarine...) sont le colza, le tournesol non oléique et oléique, la palme, le soja, l'olive, l'arachide, le maïs. Les huiles les plus riches en LA $(>50 \%)$ qui n'ont pas ou peu d'ALA sont le tournesol non oléique (linoléique) et le maïs; le colza est la plus riche en ALA ( $9 \%$ ) et son rapport LA/ALA est le plus bas $(2,5)$. Les consommations apparentes à l'échelle française sont 
Tableau 1. Estimation des produits non utilisés non consommables ou consommables (gaspillage) pour des hypothèses de pertes minimales.

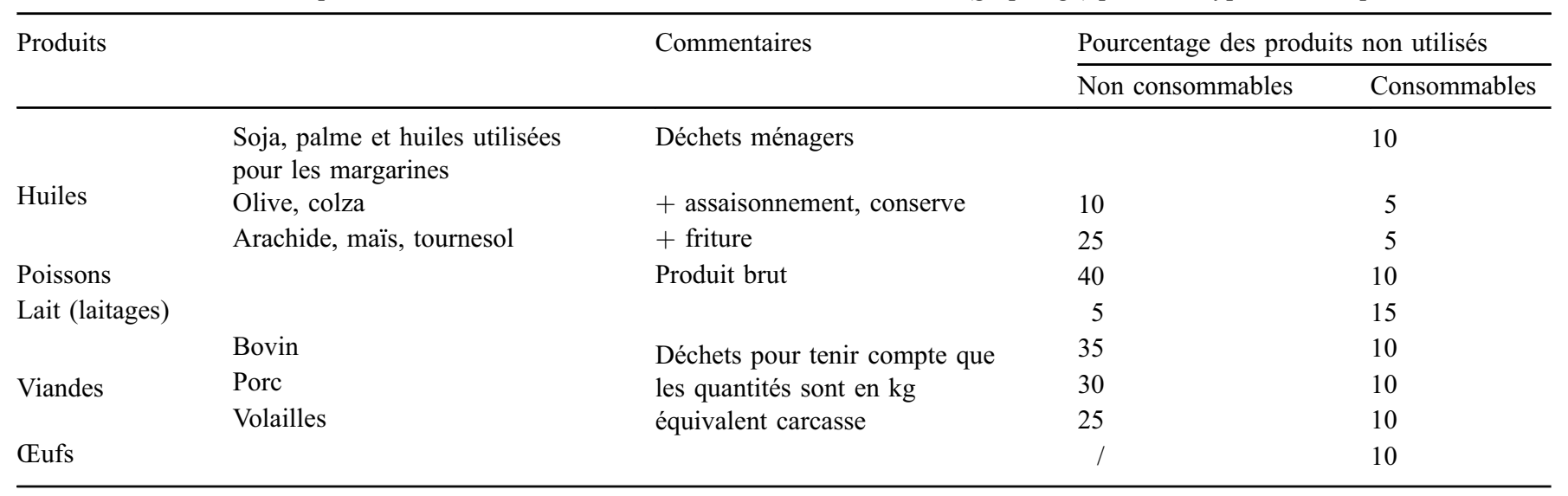

connues, sauf pour le tournesol oléique non distingué dans les statistiques, alors qu'il a une faible teneur en LA (15 au lieu de $68 \%$ ). La culture du tournesol oléique a commencé en 2000 pour atteindre en 2010 environ $50 \%$ des surfaces en France. Néanmoins, sur la base des statistiques d'importations et d'exportations (respectivement $44 \%$ et $62 \%$ de la production des années 2008-2010) et d'avis d'experts, nous avons considéré que seulement $20 \%$ de l'huile de tournesol consommée provenait de l'oléique à la fin de la période d'étude.

Une part importante des huiles n'est pas consommée, en particulier celles utilisées pour la conserverie, la partie des huiles de friture non absorbées par les aliments ainsi que l'huile de fond de poêle. Les quantités d'huiles alimentaires usagées utilisées lors des opérations de friture et destinées à l'alimentation humaine, ou utilisées en restauration commerciale et collective sont estimées entre 80000 et $170000 \mathrm{t}$ selon l'Ademe (2001), soit $11 \%$ et $24 \%$ de la consommation apparente. Nous avons classé les huiles en trois catégories pour lesquelles la fraction non consommée est supposée croissante (Tab. 1): (i) confection des margarines et de divers produits comme les pâtisseries pour lesquels il n'y a que des pertes provenant de produits non consommés, (ii) conserverie et l'assaisonnement, (iii) friture. Ces différents types de pertes correspondent à $20 \%$ de la totalité des huiles consommées. Compte tenu de la forte incertitude portant sur ces estimations, nous avons aussi considéré deux autres hypothèses : $90 \%$ et $80 \%$ de la valeur calculée, soit $27 \%$ et $35 \%$ d'huiles non consommées (Tab. 1). L'hypothèse de pertes minimales est retenue pour analyser les séries annuelles (on parlera de consommation apparente corrigée); les autres seront considérées pour calculer la composition moyenne de l'assiette à trois dates.

\subsection{Produits animaux}

\subsubsection{Quantités apparentes consommées}

Les quantités apparentes consommées par an pour le lait (avant transformation en beurre et produits laitiers), les viandes, les œufs ainsi que les poissons (Agreste, France Agrimer) ont été exprimées par jour et par personne. Les quantités de viande exprimées en équivalent carcasse ont été corrigées des pertes (gras, os), respectivement $35 \%$, 30\% et
$25 \%$ pour les bovins, porc et volailles, auxquelles s'ajoutent les pertes portant sur la non-consommation, estimées à $10 \%$ à partir de données agrégées au niveau européen (FAO, 2008). Pour le lait et les produits laitiers, les pertes ont été estimées au total à $20 \%$; celles des œufs à $10 \%$; pour les poissons et coquillages consommées entre 1998-2013, un taux de perte de $40 \%$ (parties non consommées et pertes à la consommation) (Rai et al., 2012).

\subsubsection{Composition en acides gras des produits animaux}

La composition des produits animaux en AGPI dépend beaucoup de l'alimentation animale (Anses, 2011), mais elle ne peut être reliée simplement à la nature des aliments compte tenu de la digestion et du métabolisme différents entre ruminants et monogastriques (Doreau et al., 2012). Toutefois, de nombreux travaux montrent que les différences de composition en LA/ALA de la ration se retrouvent dans la composition des produits (lait, muscle, œuf), et ce tant pour la ration de base (céréales, herbe: Tab. 1) que pour la complémentation (huiles ajoutées et huiles résiduelles des tourteaux). À titre d'exemple, le rapport LA/ALA du lait de vache est d'autant plus faible que la ration de base est composée d'herbe pâturée au lieu de maïs ensilé (Couvreur et al., 2006) et que le tourteau est à base de lin plutôt que de colza ou de soja (Szumacher-Strabel et al., 2011). En conséquence, le rapport LA/ALA du lait, du muscle ou de l'œuf diminue ou augmente en fonction de ce même rapport pour la ration de base et/ou la complémentation (Duru et Magrini, 2016).

Pour savoir si la composition des produits animaux en AGPI a évolué au cours des 20 dernières années, nous avons calculé la composition moyenne des rations à partir de leur teneur en LA et ALA, en distinguant les huiles, ainsi que les prairies et céréales pour lesquelles le rapport LA/ALA varie de 0,3 (herbe) à 28 (maïs grain); les valeurs pour le blé, l'orge et le maïs ensilage étant intermédiaires $(11,2 ; 8,2 ; 6)$. Les quantités de matières premières utilisées pour l'alimentation des animaux sont exprimées par personne pour tenir compte de l'augmentation de la population au cours de la période d'étude. Pour comparer les quantités de LA et ALA consommées selon notre méthode à celles basées sur des enquêtes au cours de la période 2006-2010, nous avons calculé la composition 
Tableau 2. Teneurs (moyenne, minimale et maximale) en LA et ALA des produits animaux (en mg/100 g de produit).

\begin{tabular}{llrrrl}
\hline Produit & Ration & \multicolumn{1}{c}{ LA } & \multicolumn{1}{c}{ ALA } & LA/ALA & Références $^{1}$ \\
\hline \multirow{2}{*}{ Lait } & Herbe & $82(58-89)$ & $28(19 ; 32)$ & 2,9 & \\
& Maïs & $73(60-90)$ & $12,5(8-15)$ & 5,9 & Couvreur et al., 2006; Borreani et al., 2013 \\
Viande rouge & Herbe & $75(52-120)$ & $35(21-60)$ & 2,2 & Pighin et al., 2016; Razminowicz et al., 2006; \\
Porc & Maïs & $118(73-170)$ & $16(10-24)$ & 7,4 & van Elswyk et McNeill, 2014 \\
Volaille & & $450(400-500)$ & $42(30-50)$ & 10,7 & Turner et al., 2014; Kartikasari et al., 2012 \\
Euf & & $250(150-300)$ & $20(10-25)$ & 12,5 & Antruejo et al., 2011 \\
Poisson gras & $250(140-350)$ & $25(10-35)$ & 10 & Karsten et al., 2010 \\
Poisson maigre & & 310 & 170 & 1,8 & Ciqual \\
& & 190 & 30 & 6,3 & \\
\hline
\end{tabular}

ALA : acide alpha-linolénique; LA : acide linoléique.

${ }^{1}$ Mourot, 2015 (la plupart des produits) et Butler, 2014 pour l'ensemble des produits au Royaune-Uni.
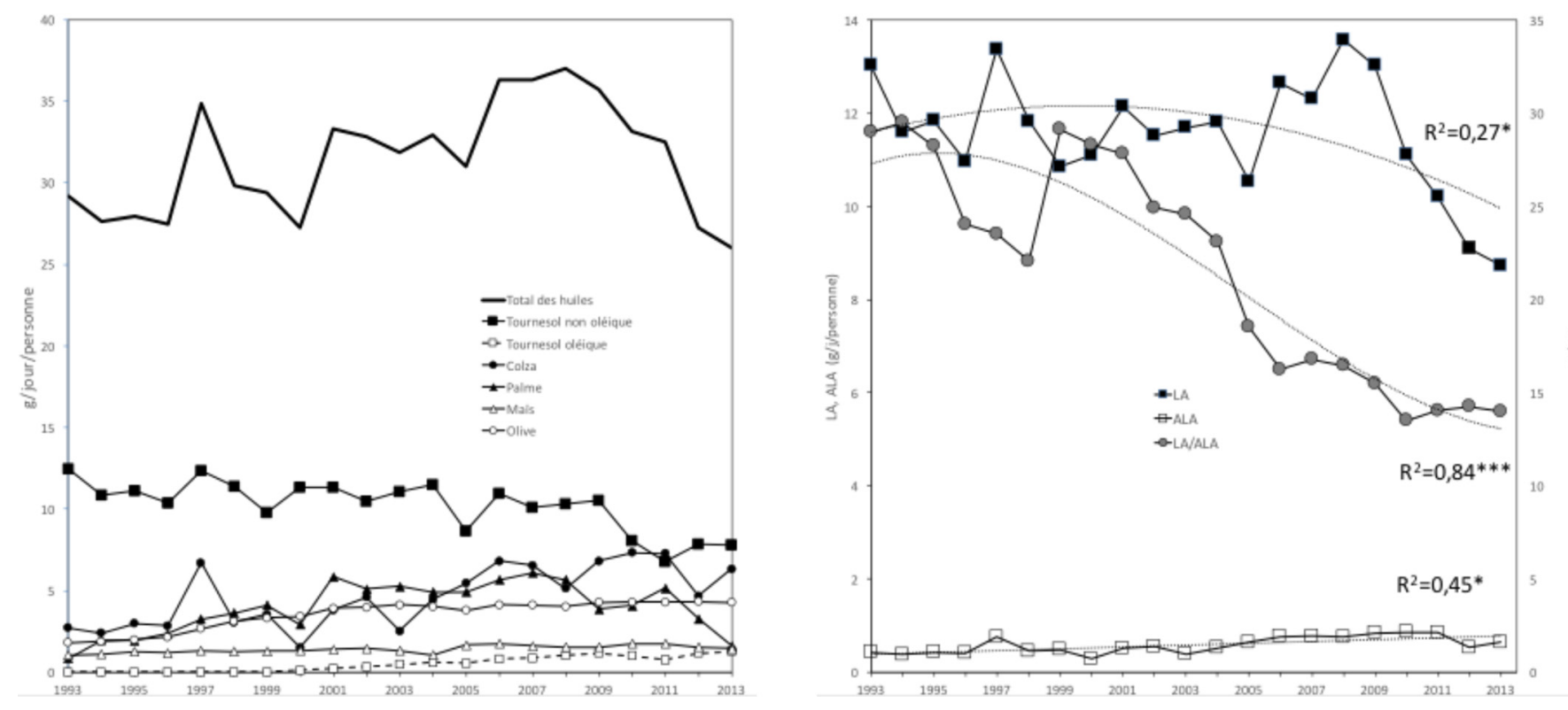

Fig. 1. Évolution de la consommation apparente corrigée des pertes ( $c f$. Tab. 1) des principales huiles végétales et des margarines : (a) quantités (source: Agreste); (b) acides linoléique (LA) et alpha-linolénique (ALA) (g par jour et par personne); les courbes de tendance (en pointillées) et coefficients de détermination sont indiqués sur la Figure $1 \mathrm{~b}$.

moyenne du produit (lait ou viande) en tenant compte de la proportion des différents systèmes d'alimentation des animaux dans l'offre de produits (Devun et Guinot, 2012). Pour l'élevage laitier, les volumes de lait produit sont de $58 \%$ pour le système d'alimentation à base de maïs, $20 \%$ pour le système mixte et $22 \%$ pour le système herbager (la part d'herbe étant respectivement de $20 \%, 45 \%$ et $90 \%$ ). Pour les viandes rouges (bovin, ovin), un tiers du volume provient des vaches de réforme des troupeaux laitiers (même alimentation que pour les vaches laitières) (IDELE, 2011), et nous estimons que les deux autres tiers de ce volume proviennent de races à viande pour lesquelles l'engraissement comprend une part importante d'herbe ou une alimentation à base de maïs et de concentrés (Duru et Magrini, 2016). Les données indiquées dans le Tableau 2 sont des moyennes pour un animal entier. Pour les poissons, nous avons considéré la base Ciqual en distinguant les poissons gras et maigres, ainsi que les coquillages et crustacés.

\section{Résultats}

\subsection{Tendances d'évolution par catégorie de produits}

\subsubsection{Huiles végétales et margarines}

Entre 1993 et 2008, la consommation apparente d'huiles par personne a augmenté, passant d'un peu moins de $30 \mathrm{~g} / \mathrm{j}$ à presque $40 \mathrm{~g} / \mathrm{j}$, puis elle a baissé en revenant à sa valeur initiale en 2013 (Fig. 1a). Les évolutions marquantes sont la réduction des huiles de tournesol non oléique et d'arachide au profit des huiles de colza et d'olive dont les consommations ont plus que doublé au cours de la période étudiée. À partir de 2008, la consommation de LA a diminué, surtout du fait d'une moindre utilisation des huiles de tournesol et de palme. La quantité de LA a diminué de 12 à $9 \mathrm{~g} / \mathrm{j}$, et celle de ALA est passée de 0,38 à $0,65 \mathrm{~g} / \mathrm{j}$. Avant 2008 , l'augmentation continue des quantités d'huiles consommées n'a pas empêché une diminution du 

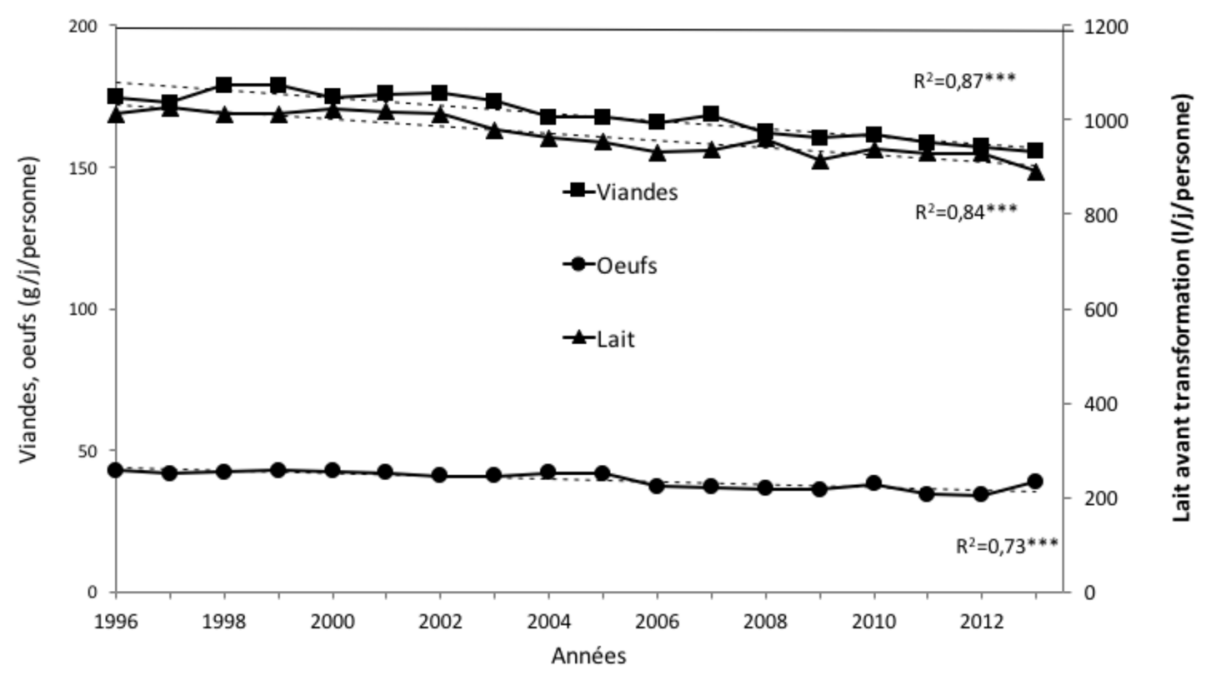

Fig. 2. Évolution des consommations apparentes en viandes (équivalent carcasse), œufs (ordonnée de gauche) et lait (avant transformation en laitages) (ordonnée de droite) (source: Agreste); les courbes de tendance sont indiquées en pointillés.

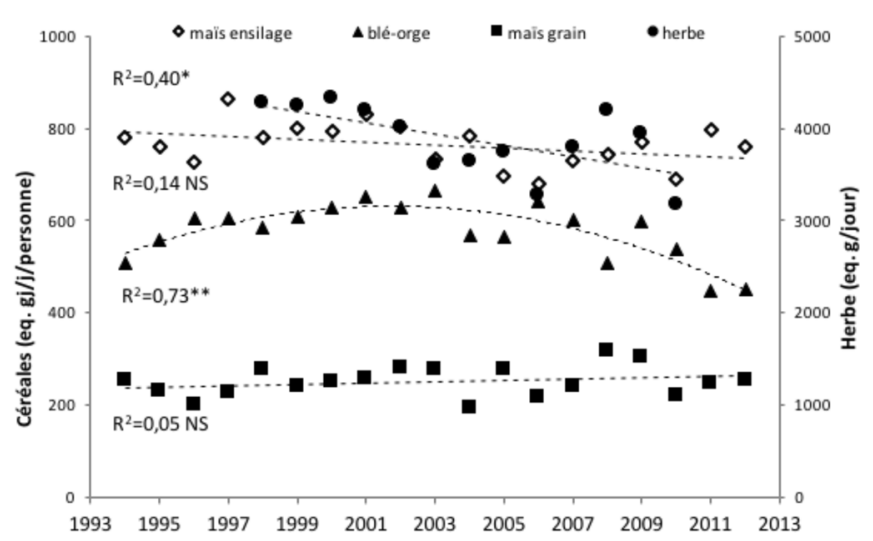

Fig. 3. Apport de céréales (maïs grain et ensilage de maïs, blé et orge) et d'herbe à l'alimentation des animaux (valeurs rapportées à une personne); source Agreste; les courbes de tendance (en pointillés) et coefficients de détermination sont indiqués.

rapport LA/ALA du fait d'une substitution entre huiles de composition différente. En conséquence, le rapport LA/ALA diminue régulièrement de 30 à 14 à partir de 1998 (Fig. 1b).

\subsubsection{Produits animaux}

\subsubsection{Quantités apparentes consommées}

La consommation apparente des produits animaux, tant carnés que laitiers, a régressé significativement au cours des deux dernières décennies (Fig. 2). La consommation apparente de lait (avant transformation en produits laitiers) est passée de $960 / \mathrm{j}$ en 1996 à $845 \mathrm{~g} / \mathrm{j}$ en 2013 et celle produits carnés de $175 \mathrm{~g} / \mathrm{j}$ en 1996 à $156 \mathrm{~g} / \mathrm{j}$ en 2013 . Les consommations de viandes de bœuf et de porc ont baissé alors que la consommation de volaille a augmenté. La consommation d'œufs est par contre restée relativement constante (un peu moins de $50 \mathrm{~g} / \mathrm{j} /$ personne).

\subsubsection{Composition des produits animaux en AGPI}

Les ressources fourragères ayant le rapport LA/ALA le plus élevé ont diminué (maïs ensilage) ou augmenté (maïs grain), mais de manière non significative. La contribution de l'herbe (rapport LA/ALA le plus faible) a diminué significativement et l'apport de blé et d'orge (rapport LA/ALA intermédiaire) est passé par un maximum au début des années 2000 (Fig. 3). En considérant que l'herbe et l'ensilage de maïs sont dédiés aux ruminants et les céréales aux monogastriques, on peut inférer que la composition en ALA des produits animaux s'est plutôt dégradée pour les ruminants (réduction de la part de l'herbe), alors que celle des monogastriques se serait d'abord améliorée (la part du blé et de l'orge augmentant au détriment de celle du maïs grain jusqu'au début des années 2000), mais l'inverse est observé par la suite.

Les quantités totales de tourteaux et d'huiles utilisées en alimentation animale augmentent brutalement entre 2000 et 

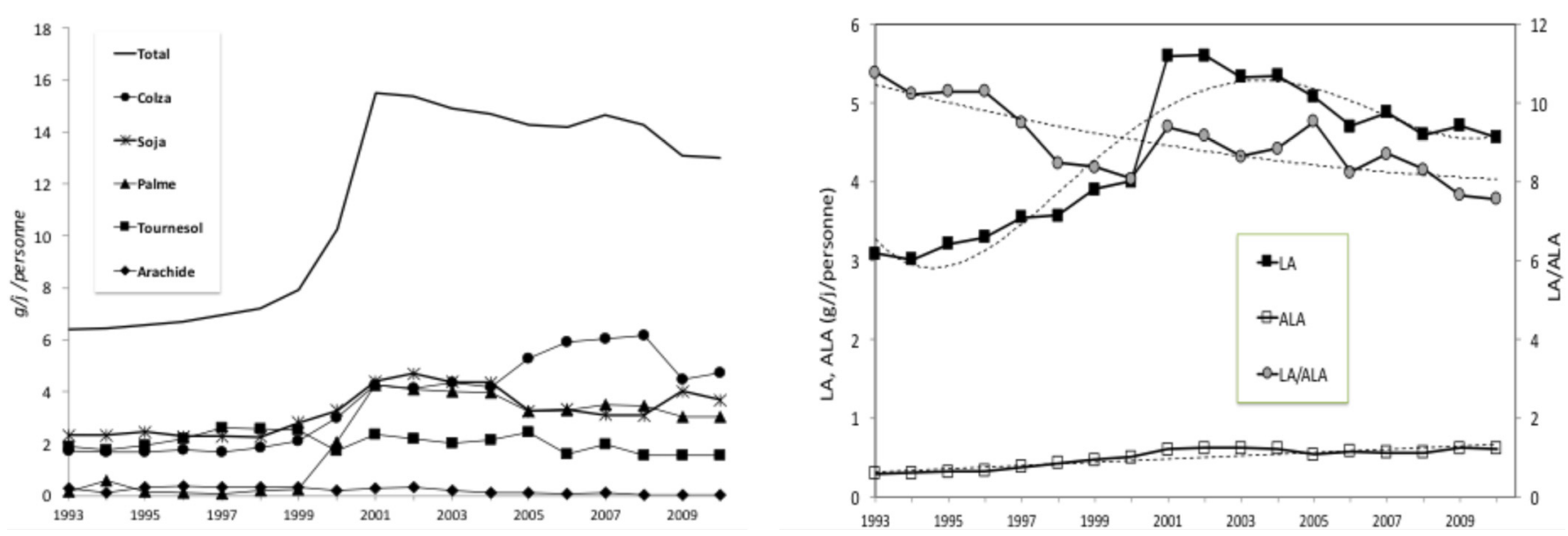

Fig. 4. Apport d'huiles (huiles des tourteaux et huiles ajoutées) dans l'alimentation animale: (a) quantités (source: Agreste); (b) acides linoléique (LA) et alpha-linolénique (ALA); valeurs rapportées à une personne. Les courbes de tendance (en pointillés) et coefficients de détermination sont indiqués sur la Figure $4 \mathrm{~b}$.

Tableau 3. Composition de l'assiette en LA et ALA ( $\mathrm{g} / \mathrm{j} /$ personne) selon les hypothèses retenues de pertes d'huiles et de composition des produits animaux.

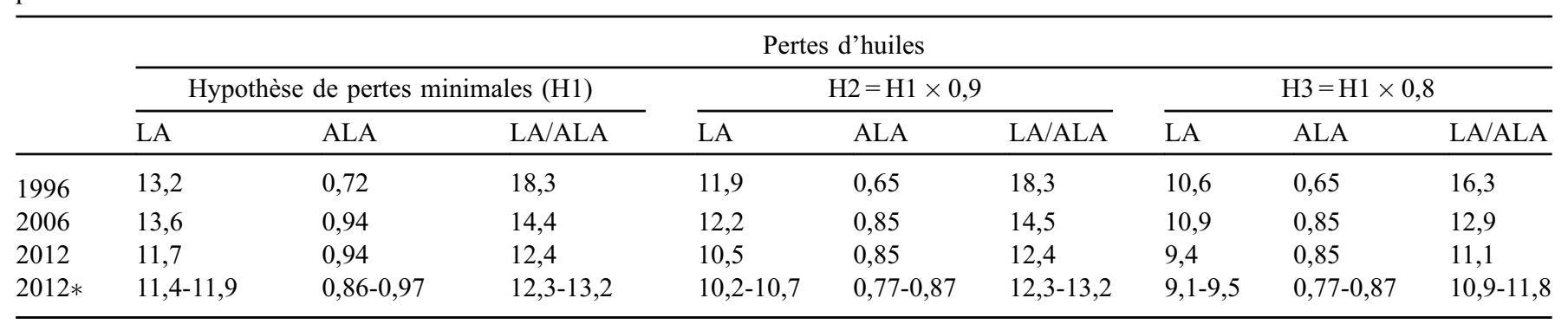

* : calculs supplémentaires pour l'année 2012 avec les valeurs mini et maxi de composition des produits animaux indiquées dans le Tableau 2.

2003 surtout du fait de l'huile de palme et du tourteau de soja (Fig. 4a). En conséquence, les apports de LA montrent un pic au début des années 2000. Néanmoins, sur l'ensemble de la période d'étude, l'apport d'ALA augmentant plus rapidement que l'apport de LA, du fait de la forte augmentation des tourteaux de colza, le rapport LA/ALA diminue régulièrement (Fig. 4b). On observe donc une forte amélioration de l'équilibre entre LA et ALA pour les concentrés.

Considérant ensemble la ration de base et les concentrés, on ne peut donc pas conclure à une évolution très marquée de la composition en LA et ALA des produits animaux au cours de la période étudiée. Il y a eu vraisemblablement une légère dégradation $\mathrm{du}$ fait d'une moindre contribution de l'herbe (Fig. 3), mais une amélioration par les apports de tourteaux et d'huiles de colza (Fig. 4b). On peut en déduire que l'apport en ALA par les produits animaux a vraisemblablement augmenté.

\section{2 Évolution de la composition de l'assiette à trois dates}

Les quantités d'ALA et de LA pour les huiles et les produits animaux consommées ont été calculées à trois périodes où la comparaison avec d'autres méthodes est possible. Les quantités d'huiles et de produits animaux consommées sont celles indiquées sur les Figures 1 et 2, corrigées d'une estimation des pertes (Tab. 1). Pour la composition des produits animaux, nous avons en outre retenu des valeurs moyennes, mais aussi maxi et mini indiquées dans le Tableau 2. Les apports de LA et ALA par les poissons, coquillages et crustacées ont été estimés par les données annuelles (France Agrimer), corrigées des parties non consommées, soit environ $19,5 \mathrm{~kg}$ sur l'ensemble de la période.

Quelle que soit l'hypothèse de perte pour les huiles, on observe une diminution importante du rapport LA/ALA entre les trois dates (Tab. 3), du fait d'une réduction de l'apport de LA et d'une augmentation de l'apport de ALA entre 1996 et 2006. La prise en compte de l'incertitude quant à la composition des produits animaux montre des variations inférieures à $10 \%$ quel que soit le critère (voir illustration pour 2012). Sur la base des quantités de produits animaux consommées (Fig. 3) et de leur composition moyenne (Tab. 3), les apports en LA et ALA par les produits animaux sont de 1,2 et $0,21 \mathrm{~g} / \mathrm{j} /$ personne, soit $10 \%$ et $23 \%$ des apports totaux. Les apports en ALA par les produits animaux ayant vraisemblablement légèrement augmenté (Fig. 3 et 4), on peut en conclure que l'amélioration notée entre 1996 et 2012 est sous-estimée. 


\section{Discussion}

\subsection{Comparaison des résultats avec d'autres méthodes}

Nos estimations de LA et ALA, calculées à partir des matières premières agricoles consommées et d'estimations de pertes et de composition des produits, ont été comparées à une autre méthode d'estimation indirecte, à des estimations directes de quantités d'aliments consommées, ainsi qu'à la composition du lait maternel.

\subsubsection{Consommation d'AGPI par une méthode indirecte sur le long cours}

Des estimations de consommation apparente établies par la FAO pour la France sur une longue période (1961-2012) (Duru, non publié; Schmidhuber, 2007) montrent une augmentation du rapport oméga-6/oméga-3 jusque vers les années 2000 puis une réduction ensuite (données non présentées). Cette dynamique est due à une augmentation plus importante des quantités d'oméga-6 que d'oméga-3 consommées jusqu'à la fin des années 1990, puis plus lente ensuite. Le rapport oméga-6/oméga-3 passe par un maximum de 13 dans les années 2000 puis diminue jusqu'à 11 en 2012. S'agissant des valeurs d'omégas 6 et 3 et non de LA et ALA, sans prise en compte de pertes, les données doivent être comparées avec précaution. En outre, la base de la FAO utilisée pour calculer les consommations ne reflète pas forcément la composition des aliments consommés en France. Néanmoins, la comparaison de ces données à nos estimations à partir de 1996 montre une même tendance : augmentation de l'ALA et des oméga-3, et diminution des rapports LA/ALA et oméga-6/ oméga-3.

\subsubsection{Enquêtes auprès de consommateurs}

Comparés aux études Inca 2 (Anses, 2015) (données recueillies entre 2006-2007) et Agralid (Ferry et al. com pers; données recueillies en 2010), la composition des produits étant calculée à partir de la base Ciqual, nos résultats sont similaires pour l'ALA $(0,9 \mathrm{~g} / \mathrm{j})$, mais inférieurs pour le LA. Alors que la quantité de LA consommée est de $8,4 \mathrm{~g} / \mathrm{j}$ (Anses, 2015) et de 10,2 g/j (Agralid), notre estimation est de $11,7 \mathrm{~g} / \mathrm{j}$ (2012) et 13,2 g/j (2006). En conséquence, le rapport LA/ALA calculé $(12,4$ et 14,4$)$ est plus élevé que celui établi dans les études citées $(9,6$ et 11,3$)$. Considérer des pertes d'huiles supérieures à l'hypothèse de pertes minimales (Tab. 3) ne change pas l'écart aux recommandations. Les valeurs trouvées dans l'étude Agalid s'inscrivent presque dans la fourchette de nos estimations de LA, ALA, LA/ALA lorsqu'on considère l'hypothèse de perte la plus élevée pour les huiles et l'incertitude de composition des produits animaux.

La comparaison de nos données de 2006 avec celles de l'étude INCA2 permet d'examiner plus en détail les quantités estimées pour les produits animaux. Au total, ces apports sont équivalents pour l'ALA (environ $0,19 \mathrm{~g} / \mathrm{j}$ ) et le LA ( 1,5 contre $1,4 \mathrm{~g} / \mathrm{j}$ d'après nos calculs). Cependant, la comparaison par produit montre des différences importantes pour les œufs et les viandes. Pour les œufs, la différence provient surtout de quantités de LA inférieures du fait d'une grosse différence du rapport LA/ALA : 19 (base Ciqual) vs 10 (nos données). Pour l'ensemble des viandes, nos calculs montrent des apports journaliers moitié moindre tant pour le LA $(0,55$ au lieu de $1,1 \mathrm{~g} / \mathrm{j}$ ) que 1'ALA (Anses, 2015). Pour obtenir des résultats similaires entre méthodes, il faudrait doubler les teneurs des viandes en LA et ALA. Prendre en compte les valeurs de composition des produits les plus élevées de la littérature synthétisées dans le Tableau 2 ne reviendrait à augmenter les quantités de LA et de ALA que de $20 \%$. Une hypothèse serait une sur estimation des quantités de viande consommées par enquêtes du fait d'une sous-estimation de la fraction non consommée (gaspillage ou partie non consommable : os, gras). Ne pouvant vérifier cette hypothèse, nous ne sommes donc pas en mesure d'expliquer ces écarts d'estimation entre les deux méthodes.

\subsubsection{Lait maternel}

Nous avons aussi comparé nos résultats à la teneur du lait maternel en AGPI. En effet, le lait maternel est un bon marqueur de notre alimentation (Combe et Vaysse, 2011). Toutefois, l'ALA étant peu incorporé dans les triacylglycérols, il est retrouvé en moindre quantité que le LA. Pour autant, le rapport LA/ALA est deux fois plus élevé dans le lait maternel qu'il ne l'est dans l'alimentation de la mère (Guesnet et al., 1997, 2009).

Dans plusieurs pays européens (France, Allemagne, Espagne), le rapport LA/ALA du lait maternel croît entre les périodes 1980/1990 et 1990/2005 (Ailhaud et al., 2006), et ce de manière cohérente avec la base de données de la FAO (Schmidhuber, 2007). En France, sur la base des données provenant de plusieurs régions disponibles à trois dates (1997, 2007, 2014), la composition du lait maternel en pourcentage des acides gras totaux passe de 0,57 à 0,9 d'ALA et de 13,5 à 10,5 de LA entre 1997 et 2014 , de telle sorte que le rapport LA/ALA diminue de 26 à 13; l'évolution observée étant plus rapide entre 1997 et 2007 qu'entre 1997 et 2014 (Coüdelo et al., 2014; Vaysse et al., 2011). Nos estimations de consommations apparentes de LA et ALA et les données de composition du lait maternel montrent donc de manière convergente une diminution du rapport LA/ALA au cours des 20 dernières années.

\subsection{Une composition de notre assiette en AGPI qui s'améliore mais qui reste éloignée des recommandations}

Pour les années 2006-2010, la comparaison de la composition moyenne de l'assiette en LA et ALA, à partir des consommations apparentes corrigées et des estimations par enquêtes, montre des ordres de grandeur similaires. Cette cohérence s'observe malgré des imprécisions pour les deux méthodes. La limite des méthodes par enquête tient à l'estimation visuelle des quantités consommées par le consommateur enquêté pouvant donner lieu à des sousdéclarations (Anses, 2015). Dans notre cas, une limite tient à ce que la consommation est ramenée à la personne, sans distinguer adultes et enfants. En outre, les différents taux de pertes comportent une grande incertitude tant pour les fractions non consommables que pour le gaspillage. Néanmoins, le rapport LA/ALA peut être considéré comme fiable car peu 
dépendant des niveaux de pertes considérés. Rappelons enfin que nous n'avons pas considéré les acides gras à très longue chaîne qui sont présents surtout dans les poissons gras, et dans une moindre mesure les produits animaux mais sont absents dans les huiles.

$\mathrm{Au}$ cours des 20 dernières années, nous observons une réduction $\mathrm{du}$ rapport $\mathrm{LA} / \mathrm{ALA}$ pour les trois méthodes considérées (nos estimations, la méthode indirecte de la FAO et la teneur du lait maternel). L'évolution du rapport LA/ALA peut s'analyser comme suit: d'abord une période de détérioration (1960 à 1995-2000), notamment du fait d'une réduction de la place de l'herbe dans l'alimentation des animaux (Duru et Magrini, 2016), suivie d'une période d'amélioration plus par l'augmentation de la consommation d'ALA (augmentation de la consommation d'huile de colza) que par une réduction de la consommation de LA. Malgré tout, les apports d'ALA sont toujours très insuffisants au regard des recommandations $(0,9 \mathrm{~g} / \mathrm{j}$ au lieu de 2,2 g/j recommandé), et le rapport LA/ALA est au moins égal à 9 au lieu de 4 recommandé). Les données concernant la composition du lait maternel confirment bien cette tendance. Le fait qu'avec des méthodes très différentes on obtienne des résultats convergents permet de conforter les conclusions. Malgré une augmentation de la consommation en ALA au cours des 20 dernières années, notre alimentation reste en moyenne très en deçà des recommandations.

\subsection{Facteurs ayant changé la composition en AGPI de notre assiette}

La réduction du rapport LA/ALA par augmentation de l'ALA peut être analysée comme résultant d'une diversité d'actions au sein du régime socio-technique. Le régime sociotechnique (Geels, 2004) désigne l'ensemble du système de production et d'échange formé par les choix des industriels et autres opérateurs des filières, les préférences des consommateurs, les technologies en place, l'état des connaissances des acteurs et les règles d'action collective, notamment liées aux politiques publiques. Afin de mieux comprendre les facteurs ayant changé la composition en AGPI de notre assiette, nous avons conduit en 2014 et 2015, une vingtaine d'entretiens semi-directifs avec des acteurs représentatifs des filières et des institutions. Nous synthétisons ci-après les principaux facteurs de changement du rapport LA/ALA au regard des politiques publiques, des industries agroalimentaires, des lobbies et le comportement des consommateurs.

\subsubsection{Les politiques publiques}

Liées à la santé. À l'initiative du ministère de l'Alimentation, de l'Agriculture et de la Pêche, des acteurs du régime socio-technique (producteurs, industriels, distributeurs, restauration collective, représentants des consommateurs, instances scientifiques, experts $a d$ hoc...) ont pu faire un état des lieux sur les possibles réductions des teneurs en lipides des aliments. Ces instances de concertation ont permis aux pouvoirs publics de décider de la mise en place de recommandations, de dispositifs d'incitation à l'amélioration, d'outils de suivi et d'évaluation. Elles ont aussi permis de construire une vision partagée afin de mobiliser les opérateurs dans une démarche de progrès. Un résultat important a été l'élaboration de la charte du PNNS (2009), à partir de laquelle ont été développées des campagnes d'information pour le consommateur, et des incitations auprès des industriels pour réduire les teneurs en acides gras saturés (huile de palme) ou augmenter les teneurs en oméga-3 (Souillac et al., 2010). Grâce à l'abrogation du décret de 1973 sur l'utilisation des huiles en friture, des huiliers ont pu communiquer sur l'huile de colza dont le rapport LA/ALA est faible. Certains fast-foods communiquent sur la qualité des huiles utilisées en fritures. Certaines marques de margarine enrichissent leur produit en oméga-3.

Liées à la production agricole. Les incitations à la production de cultures énergétiques, notamment à partir du colza (Thomas et al., 2013), ont indirectement permis de réduire l'usage des tourteaux de soja. La directive 2003/30/CE a en effet prévu que les biocarburants représentent $2 \%$ des carburants consommés pour les transports dans l'UE en 2005 , et $5,75 \%$ en 2010 . Ces politiques publiques ont ainsi indirectement impacté la composition des produits animaux en AGPI: la part du colza relativement au soja dans les concentrés est passée de $33 \%$ à $65 \%$ au cours de la période d'étude, et il a été montré que le remplacement du tourteau de soja par du tourteau de colza augmente d'environ $20 \%$ la teneur en ALA du muscle (Sami et al., 2010) et du lait (Rutkowska et al., 2015). Cependant, un plafond d'utilisation du colza est sans doute atteint car compte tenu de son empreinte environnementale élevée, le parlement européen a plafonné en septembre 2013 les agrocarburants de première génération à $6 \%$. En revanche, la $\mathrm{PAC}$ n'a pas réussi à enrayer la diminution des surfaces en prairies pour l'élevage (Catan, 2014) réduisant une voie importante d'amélioration en ALA des produits des ruminants.

\subsubsection{Les stratégies des industries agroalimentaires}

Les fabricants d'aliments du bétail, tout autant que les acteurs de l'agroalimentaire, ont modifié les huiles utilisées à partir des années 2000 : réduction de l'huile de palme et augmentation de l'huile de colza. L'huile de tournesol oléique est utilisée en mélange pour fournir des huiles équilibrées pour l'alimentation humaine. On peut cependant s'étonner qu'on ne puisse la trouver pure dans la consommation courante alors qu'elle est à juste titre identifiée par les huiliers comme un atout pour les besoins de cuisine à haute température (Tressou et al., 2016). Enfin, réduire l'utilisation de l'huile de tournesol linoléique, la plus riche en LA, apparaît comme un levier important pour diminuer les apports en cet AGPI pour les consommateurs dont la consommation dépasse les recommandations.

\subsubsection{Comportement des consommateurs}

Les consommateurs par leur acte d'achat peuvent influer sur la composition de leur assiette. Les campagnes organisées contre l'huile de palme ont vraisemblablement influencé les industriels pour utiliser des huiles plus appréciées du consommateur. Les consommateurs pourraient aussi privilégier les huiles de colza ou de noix au détriment des huiles de tournesol et de maïs. Pour les produits animaux, il reste cependant difficile de choisir les produits en fonction des modes d'élevage, excepté pour les produits issus de la filière 
certifiée Bleu Blanc Cœur garantissant une teneur en ALA plus élevée que les mêmes produits non labellisés (Tressou et al., 2016), notamment grâce à l'apport de lin aux animaux. Une autre possibilité, mais avec moins de garantie sur la teneur en ALA, est de choisir des laitages et viandes rouges mentionnant une utilisation importante de l'herbe dans leur cahier des charges, tel qu'en agriculture biologique ou dans nombre d'AOP fromagères (Duru et Magrini, 2016).

\section{Conclusion}

Nous avons estimé la consommation apparente (statistiques de production, corrigées des fractions non consommables, des déchets et des gaspillages) de LA et ALA apportés par les huiles et les produits animaux sur une vingtaine d'années. Compte tenu de la composition des huiles utilisées, nous avons montré une tendance à l'amélioration (augmentation de l'ALA et légère réduction du LA). Pour les produits animaux, cette tendance à l'amélioration est aussi liée à l'utilisation d'huiles ou de tourteaux plus riches en ALA. Nos évaluations sont cohérentes avec les résultats d'autres méthodes: enquête de déclaration par le consommateur, composition du lait maternel. Les valeurs moyennes observées restent encore très éloignées des recommandations, quelle que soit la méthode d'estimation. D'un point de vue méthodologique, soulignons que différentes hypothèses de composition de produits animaux ou de pertes d'huiles ne changent pas fortement l'écart observé aux recommandations.

Les changements incrémentaux permis par les nouvelles politiques publiques et les stratégies des industriels de ces dernières années restent donc insuffisants. Seul un changement de grande ampleur dans les modes d'alimentation des animaux et le choix d'huiles conformes aux recommandations permettront au consommateur d'accéder à une alimentation plus équilibrée en oméga- 3 et -6 pour une meilleure santé.

Conflits d'intérêt. Les auteurs déclarent ne pas avoir de conflits d'intérêt.

\section{Références}

Ademe. 2001. Gestion des déchets des commerces de la restauration - Réf. 4064.

Agreste. http://www.agreste.agriculture.gouv.fr/.

Agrimer. http://www.franceagrimer.fr/.

Ailhaud G, Massiera F, Weill P, Legrand P, Alessandri JM, Guesnet P. 2006. Temporal changes in dietary fats: role of $n-6$ polyunsaturated fatty acids in excessive adipose tissue development and relationship to obesity. Progr Lipid Res 45: 203-236.

Anses. 2011. Actualisation des apports nutritionnels conseillés pour les acides gras. Rapport d'expertise collective. https://www.anses. $\mathrm{fr} / \mathrm{fr}$.

Anses. 2015. Actualisation des apports nutritionnels conseillés pour les acides gras. Rapport d'expertise collective. https://www.anses. $\mathrm{fr} / \mathrm{fr}$.

Antruejo A, Azcona JO, Garcia PT, Gallinger C, Rosmini M, Ayerza R. 2011. Omega-3 enriched egg production: the effect of $\alpha$-linolenic $\omega-3$ fatty acid sources on laying hen performance and yolk lipid content and fatty acid composition. Br Poultry Sci 52: 750-760.
Borreani G, Coppa M, Revello-Chion A, Comino L, Giaccone D, Ferlay A. 2013. Effect of different feeding strategies in intensive dairy farming systems on milk fatty acid profiles, and implications on feeding costs in Italy. J Dairy Sci 96: 6840-6855.

Bousquet M, Calon F, Cicchetti F. 2011. Impact of $\omega-3$ fatty acids in Parkinson's disease. Ageing Res Rev 10: 453-463.

Catan A. 2014. La préservation des prairies dans la PAC: les raisons d'une illusion. Courrier de l'Environnement 64: 91-104.

Ciqual. https://www.data.gouv.fr/.../table-ciqual-de-compositionnutritionnelle-des-aliments-f....

Colussi G, Catena C, Novello M, Bertin N, Sechi LA. 2017. Impact of omega-3 polyunsaturated fatty acids on vascular function and blood pressure: relevance for cardiovascular outcomes. Nutr Metab Cardiovas 27: 191-200.

Combe N, Vaysse C. 2011. Teneurs en acides gras polyinsaturés essentiels du lait maternel en France: évolution des teneurs en acides linoléique et alpha-linolénique. Med \& Nutr 47: 5-9.

Couëdelo L, Billeaud C, Lamireau D, Perez P, Rigourd V, Buffin R. 2014. Evolution of essential fatty acid composition of French breast milk from 1997 to 2014. EFL Montpellier (poster).

Couvreur S, Hurtaud C, Lopez C, Delaby L, Peyraud JL. 2006. The linear relationship between the proportion of fresh grass in the cow diet, milk fatty acid composition, and butter properties. J Dairy Sci 89: 1956-1969.

Delgado-Lista J, Perez-Martinez P, Lopez-Miranda, J, Perez-Jimenez F. 2011. Long chain omega-3 fatty acids and cardiovascular disease: a systematic review. Br J Nutr 107: S201-S213.

Devun J, Guinot C. 2012. Alimentation des bovins : rations moyennes et autonomie alimentaire. Techniques 40 .

Doreau M, Fievez V, Troegeler-Meynadier A, Glasser F. 2012. Métabolisme ruminal et digestion des acides gras longs chez le ruminant: le point des connaissances récentes. Prod Anim 25: 361-374.

Duru M, Magrini M. 2016. Quel potentiel de la prairie pour équilibrer notre alimentation en acides gras ? Fourrages 228: 301-312.

FAO. 2008. Graisses et acides gras dans la nutrition humaine. Rapport d'une consultation d'experts. FAO.

Geels FW. 2004. From sectoral systems of innovation to sociotechnical systems: Insights about dynamics and change from sociology and institutional theory. Res Policy 33: 897-920.

Guesnet P, Alasnier C, Alessandri JM, Durand G. 1997. Modifying the $\mathrm{n}-3$ fatty acid content of the maternal diet to determine the requirements of the fetal and suckling rat. Lipids 32 (5): 527-535.

Guesnet P, Combe N, Ailhaud G, Alessandri LM. 2009. La teneur en acides gras polyinsaturés du lait maternel: un marqueur biologique fiable du niveau de consommation des populations. OCL 16 (1): 1-3.

IDELE. 2011. La production de viande bovine en France. Qui produit quoi, comment et où ? 145: $60 \mathrm{p}$.

Karsten HD, Patterson PH, Stout R, Crews G. 2010. Vitamins A, E and fatty acid composition of the eggs of caged hens and pastured hens. Renew Agricult Food Syst 25: 45.

Kartikasari LR, Hughes RJ, Geier MS, Makrides M, Gibson RA. 2012. Dietary alpha-linolenic acid enhances omega-3 long chain polyunsaturated fatty acid levels in chicken tissues. Prostag Leukotr Ess 87: 103-109.

Kiecolt-Glaser JK, Belury M, Andridge R, Malarkey WB, Hwang BS, Glaser R. 2012. Omega-3 supplementation lowers inflammation in healthy middle-aged and older adults: a randomized controlled trial. Brain Behav Imm 6: 988-995.

Loef M, Walach H. 2013. The omega-6/omega-3 rapport and dementia or cognitive decline: a systematic review on human studies and biological evidence. J Nutr Gerontol Geriatr 32: $1-23$. 
Molendi-Coste O, Legry, V, Leclercq IA. 2011. Why and how meet n-3 PUFA dietary recommendations? Gastroenter Res Pract, Article ID 364040, 11.

Mourot J. 2015. Évolution de la qualité des produits animaux ces cinquante dernières années. Cah Nutr Diet 50: 1-6.

Pighin D, Pazos A, Chamorro V, Paschetta F, Cunzolo S, Godoy F. 2016. Contribution of Beef to Human Health: A Review of the Role of the Animal Production Systems. Hindawi Publishing Corporation; Article ID 8681491, 10 p.

Pottel L, Lycke M, Boterberg T, Foubert I, Pottel H, Duprez F. 2013. Omega-3 fatty acids: physiology, biological sources and potential applications in supportive cancer care. Phytochem Rev 13: 223-244.

Rai AK, Swapna HC, Bhaskar N, Baskaran V. 2012. Potential of seafood industry byproducts as sources of recoverable lipids: fatty acid composition of meat and non-meat component of selected indian marine fishes. J Food Biochem 36: 441-448.

Rapport sur les lipides du Programme National Nutrition Santé. 2009. agriculture.gouv.fr/sites/minagri/files/../synthese-rapport-groupePNNS-lipides.pdf.

Razminowicz RH, Kreuzer M, Scheeder MRL. 2006. Quality of retail beef from two grass-based production systems in comparison with conventional beef. Meat Sci 3: 351-361.

Rosenblat JD, Cha DS, Mansur RB, McIntyre RS. 2014. Inflamed moods: A review of the interactions between inflammation and mood disorders. Prog Neuro-Psychoph 53C: 23-34.

Rutkowska J, Białek M, Bagnicka E, Jarczak J, Tambor K, Strzałkowska N. 2015. Effects of replacing extracted soybean meal with rapeseed cake in corn grass silage-based diet for dairy cows. J Dairy Res 82: 161-168.

Sami AS, Schuster M, Schwarz FJ. 2010. Performance, carcass characteristics and chemical composition of beef affected by lupine seed, rapeseed meal and soybean meal. J Anim Physiol Anim Nutr 94: 465-473.

Schmidhuber J. 2007. The EU diet-evolution, evaluation and impacts of the CAP. WHO Forum on "Trade and healthy food and diets", Montreal, Canada.

Simopoulos AP. 2007. Evolutionary aspects of diet, the omega-6/ omega-3 rapport and genetic variation: nutritional implications for chronic diseases. Biomed Pharmacother 60: 502-507.

Szumacher-Strabel M, Cieslak A, Zmora P, Pers-Kamczyc E, Bielińska S, Stanisz M. 2011. Camelina sativa cake improved unsaturated fatty acids in ewe's milk. J Sci Food Agric 91: 2031-2037.

Thomas A, Schneider A, Pilorgé P. 2013. Politiques agricoles et place du colza et du pois dans les systèmes de culture. Revue $A E \& S$ 3: 7 www.agronomie.asso.fr/..de..2013../revue-aes-vol3-n1-7/.

Tressou J, Pasteau S, Darrigo Dardinet S, Simon N, Le Guillou C. 2016. Données récentes sur les apports en acides gras des Français. OCL 23(3).

Turner TD, Mapiye C, Aalhus JL, Beaulieu AD, Patience JF, Zijlstra RT. 2014. Flaxseed fed pork: n-3 fatty acid enrichment and contribution to dietary recommendations. Meat Sci 96: 541-547.

van Elswyk ME, McNeill SH. 2014. Impact of grass/forage feeding versus grain finishing on beef nutrients and sensory quality: The U.S. experience. Meat Sci 96: 535-540.

Vaysse C, Billeau C, Guesnet P, et al. 2011. Teneurs en acides gras polyinsaturés essentiels du lait maternel en France: évolution des teneurs en acides linoléique et alpha-linolénique. Med Nutr 2: 5-9.

Yang LG, Song ZX, Yin H, Wang YY, Shu GF, Lu HX. 2016. Low n-6/n-3 PUFA rapport improves lipid metabolism, inflammation, oxidative stress and endothelial function in rats using plant oils as n-3 fatty acid source. Lipids 51: 49-59.

Citation de l'article : Duru M, Magrini M-B. 2017. Composition en acides gras poly-insaturés de notre assiette et utilisation des matières premières agricoles en France: une amélioration lente, mais insuffisante. OCL 24(2): A201 